\title{
A machine learning approach for genome-wide prediction of morbid and druggable human genes based on systems-level data
}

\author{
Pedro R Costa, Marcio L Acencio ${ }^{*}$, Ney Lemke \\ From 5th International Conference of the Brazilian Association for Bioinformatics and Computational Biology \\ (X-meeting 2009) \\ Angra Dos Reis, RJ, Brazil. 18-22 October 2009
}

\begin{abstract}
Background: The genome-wide identification of both morbid genes, i.e., those genes whose mutations cause hereditary human diseases, and druggable genes, i.e., genes coding for proteins whose modulation by small molecules elicits phenotypic effects, requires experimental approaches that are time-consuming and laborious. Thus, a computational approach which could accurately predict such genes on a genome-wide scale would be invaluable for accelerating the pace of discovery of causal relationships between genes and diseases as well as the determination of druggability of gene products.

Results: In this paper we propose a machine learning-based computational approach to predict morbid and druggable genes on a genome-wide scale. For this purpose, we constructed a decision tree-based meta-classifier and trained it on datasets containing, for each morbid and druggable gene, network topological features, tissue expression profile and subcellular localization data as learning attributes. This meta-classifier correctly recovered $65 \%$ of known morbid genes with a precision of $66 \%$ and correctly recovered $78 \%$ of known druggable genes with a precision of $75 \%$. It was than used to assign morbidity and druggability scores to genes not known to be morbid and druggable and we showed a good match between these scores and literature data. Finally, we generated decision trees by training the $\mathbf{J} 48$ algorithm on the morbidity and druggability datasets to discover cellular rules for morbidity and druggability and, among the rules, we found that the number of regulating transcription factors and plasma membrane localization are the most important factors to morbidity and druggability, respectively.

Conclusions: We were able to demonstrate that network topological features along with tissue expression profile and subcellular localization can reliably predict human morbid and druggable genes on a genome-wide scale. Moreover, by constructing decision trees based on these data, we could discover cellular rules governing morbidity and druggability.
\end{abstract}

\section{Background}

Currently, the large-scale experimental identification of both morbid genes, i.e. those genes whose mutations cause hereditary human diseases, and druggable genes,

\footnotetext{
* Correspondence: mlacencio@ibb.unesp.br

Departamento de Física e Biofísica, Instituto de Biociências de Botucatu UNESP - Univ Estadual Paulista, Distrito de Rubião Jr. s/n, Botucatu, São

Paulo, 18618-970, Brazil

Full list of author information is available at the end of the article
}

i.e. genes coding for proteins whose modulation by small molecules elicits phenotypic effects, demands time-consuming and laborious approaches that are impractical for rapidly revealing the causal relationships between genes and diseases and determining the druggability of gene products. The discovery of morbid genes, for instance, requires a large effort to gather inheritance patterns from families with the disease and to perform linkage and mutation analyses in order to identify

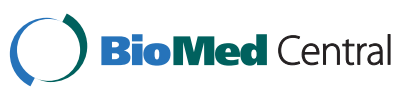

(C) 2010 Costa et al; licensee BioMed Central Ltd. This is an open access article distributed under the terms of the Creative Commons Attribution License (http://creativecommons.org/licenses/by/2.0), which permits unrestricted use, distribution, and reproduction in any medium, provided the original work is properly cited. 
candidate gene(s) involved in a particular hereditary disorder [1]. In similar fashion, the discovery of new drug targets also requires a large effort involving a variety of genomics, proteomics, genetic association and forward and reverse genetics-related techniques [2] in order to find drugs capable to modulate disease processes.

In the light of above mentioned facts, a computational approach which could accurately predict morbid and druggable genes, especially on a genome-wide scale, would be thus invaluable since the number of experimental techniques to be performed to discover these genes could be minimized. With the vast amount of current available systems-level data, such as molecular interaction data and genome-wide gene expression and subcellular localization data, we have now the opportunity for developing a computational approach based on data mining tools, such as machine learning, to extract patterns that could be used as genome-wide predictors of morbid and druggable genes. Based on this assumption, we have previously used a machine learning-based methodology as a data mining tool to extract knowledge from systems-level data and then apply this knowledge to predict essential genes on a genome-wide scale and determine cellular rules for essentiality on Escherichia coli[3] and Saccharomyces cerevisiae[4]. In addition to attain successful prediction rates, we have also obtained biologically plausible cellular rules for gene essentiality using this machine learning approach.

Due this successful prediction of essential genes and determination of cellular rules for gene essentiality in Escherichia coli and Saccharomyces cerevisiae, we sought to verify in this present work whether a similar machine learning-based approach is able to predict human morbid and druggable genes on a genome-wide scale and to reveal cellular rules governing morbidity and druggability of genes. Using knowledge acquired from network topological features, tissue expression profile and subcellular localization data, we show here that the classifiers trained on these systems-level data can reliably predict morbid and druggable genes on a genome-wide scale and also can define some general rules governing morbidity and druggability in human.

\section{Results and Discussion}

The integrated network of human gene interactions and calculation of topological features

For obtaining the network topological features used as training data for predicting morbid and druggable genes, we first constructed an integrated network of human gene interactions (INHGI) simultaneously containing experimentally verified protein physical interactions, metabolic interactions and transcriptional regulation interactions (definitions for each type of interaction are detailed in "Methods"). This network is comprised by 10,241 genes interacting with one another via 43,342 protein physical interactions, 24,540 metabolic interactions and 3,015 transcriptional regulation interactions. INHGI contains approximately $25 \%$ of the already identified $\approx 45,000$ human genes according to the EntrezGene database [5].

From the INHGI, we calculated 12 different topological features for each gene, including degree centralities for each type of interaction, clustering coefficient, betweenness centralities for each type of interaction, closeness centrality and identicalness. The detailed description of these topological features and how they were calculated are found in the Additional file 1 and "Methods".

\section{Evaluation of classifier performance}

To examine how well a machine learning-based approach is able to predict human morbid and druggable genes on a genome-wide scale using knowledge acquired from systems-level data, we designed a metaclassifier similar to that used to predict essential genes in Escherichia coli [3] and Saccharomyces cerevisiae [4] and trained it on network topological features, tissue expression profile and subcellular localization data of known morbid and druggable genes (see "Methods" for details). We then assessed its performance by measuring its median recall, precision and area under the curve (AUC) of the receiver operating characteristic (ROC) curve across 10 different normal morbidity datasets and 10 different normal druggability datasets (see "Methods" for more details).

Before analyzing the performance measures of our meta-classifier trained on the datasets described above, we decided to estimate the performance measures of our meta-classifier on equivalent normal morbidity and druggability datasets where the class labels-morbid and druggable-were randomly shuffled among genes (shuffled morbidity and shuffled druggability datasets) and then compared them with our meta-classifier trained on the normal morbidity and druggability datasets. This was done to check whether the meta-classifier trained on non-shuffled datasets learned the traits actually associated with morbidity and druggability instead of traits associated with any random subset of genes. For this comparison, we used the Wilcoxon signed-rank statistical test as described in "Methods". As can be observed in Table 1, all performance measures of our meta-classifier trained on the correspondent shuffled datasets were statistically different from measures of meta-classifier trained on normal datasets (for all performance measures, $W \leq W_{c}$ with $N=10$ at the $\mathrm{p}=0.05$ level; see "Methods" and [6]), thereby indicating that the 
Table 1 Classifier performance measures for prediction of morbid and druggable genes

\begin{tabular}{|c|c|c|c|c|c|}
\hline \multicolumn{6}{|c|}{ Prediction of morbid genes } \\
\hline Performance measure & Median $[\min , \max ]^{1}$ & Median $\left[\right.$ min,max ${ }^{1}$ & $N$ & W & $W_{c}(\text { two-tailed } p=0.05)^{2}$ \\
\hline & Normal & Shuffled & & & \\
\hline Precision & $0.658[0.648,0.679]$ & $0.495[0.473,0.522]$ & 10 & 0 & $8 *$ \\
\hline Recall & $0.648[0.632,0.657]$ & $0.502[0.471,0.521]$ & 10 & 0 & $8 *$ \\
\hline AUC & $0.716[0.706,0.729]$ & $0.498[0.462,0.526]$ & 10 & 0 & $8 *$ \\
\hline \multicolumn{6}{|c|}{ Prediction of druggable genes } \\
\hline \multirow[t]{2}{*}{ Performance measure } & Median $[\min , \max ]^{1}$ & Median $[\min , \max ]^{1}$ & $N$ & W & $W_{c}$ (two-tailed $\left.p=0.05\right)^{2}$ \\
\hline & Normal & Shuffled & & & \\
\hline Precision & $0.748[0.72,0.763]$ & $0.5[0.451,0.556]$ & 10 & 0 & $8 *$ \\
\hline Recall & $0.782[0.732,0.809]$ & $0.492[0.447,0.564]$ & 10 & 0 & $8 *$ \\
\hline AUC & $0.820[0.801,0.835]$ & $0.500[0.43,0.546]$ & 10 & 0 & $8 *$ \\
\hline
\end{tabular}

${ }^{1}$ Of 10 datasets

${ }^{2}$ According to table of critical values for $W$ in [6]

* Difference statistically significant

traits actually associated with morbidity and druggability were learned by our meta-classifier.

After confirmation that our meta-classifier trained on normal datasets was likely to learn the traits actually associated with morbidity and druggability, we aimed to analyze its performance measures. As shown in Table 1, for the genome-wide prediction of morbid genes, our meta-classifier achieved a median recall of 0.648 and a median precision of 0.658 , i.e., it correctly recovered $64.8 \%$ of known morbid genes with a precision of $65.8 \%$. Furthermore, the probability of a gene predicted as morbid belongs to the set of known morbid genes is $71.2 \%$ as indicated by the median AUC. For the genome-wide prediction of druggable genes, our meta-classifier achieved a median recall of 0.782 and a median precision of 0.748 , i.e, it correctly recovered $78.2 \%$ of known druggable genes with a precision of $74.8 \%$ (Table 1 ). Furthermore, the probability of a gene predicted as druggable belongs to the set of known druggable genes is $82.0 \%$ as indicated by the median AUC.

The moderate values for both median recall (0.648) and median precision (0.662) for genome-wide prediction of morbid genes indicate that the level of noise in the training data is high and likely associated with existence of shared common features between morbid and non-morbid genes that induced our meta-classifier to yield a moderate performance in discriminating morbid from non-morbid genes. This could be partially due to the approach used to select non-morbid genes: since it is impossible at present to compile a list of genes not known to cause any hereditary disease, we selected genes not known to be morbid, i.e., all genes in INHGI except the known morbid genes, as non-morbid genes. Thus, some of these non-morbid genes may actually be existing unknown morbid genes sharing common characteristics with the existing known morbid genes. Other contributing factor for the existence of shared common features between morbid and non-morbid genes could be the incompleteness of INHGI: Stumpf et al. [7], for example, have estimated that the size of human interactome (only protein-protein interactions) is about 650,000 interactions. Since our network contains about 43,000 protein-protein interactions, we could envisage that the values of all network topological parameters might change with the enlargement of network size and, therefore, some of the network topological parametersrelated shared common features between morbid and non-morbid might disappear as a consequence. The existence of shared common features between druggable and non-druggable genes also seems to affect the performance of our meta-classifier, but to a lesser extent: our meta-classifier achieved reliables values for the median recall (0.782) and precision (0.748) for genome-wide prediction of druggable genes (Table 1).

Despite these limitations discussed above, our metaclassifier trained on network topological features, tissue expression profile and subcellular localization data seems indeed to be a reliable predictor of morbid and druggable genes on a genome-wide scale as shown by Figures 1 and 2: the frequency distribution of known morbid and known druggable genes per intervals of morbidity and druggability scores-probabilities of classifying genes as morbid and druggable, respectively, as output by the meta-classifier (see "Prediction of novel morbid and druggable genes" and "Methods" for more details) - tend to increase as morbidity (Figure 1) and druggability (Figure 2) scores increase.

Evaluation of individual features on classifier performance We sought to verify the influence of individual features on the meta-classifier performance. To achieve this goal, we first trained our meta-classifier on normal morbidity 


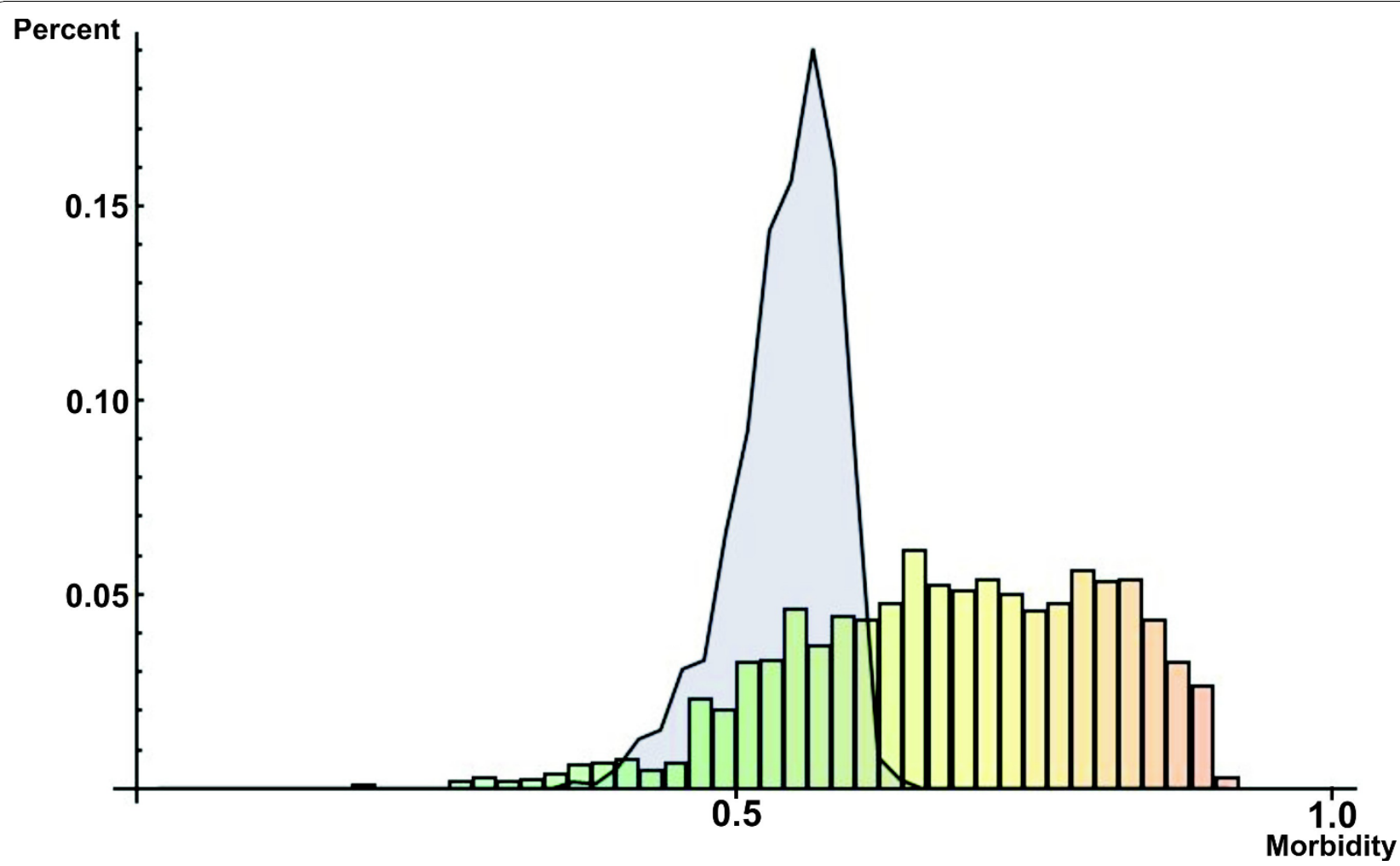

Figure 1 Frequency distribution of known morbid genes per intervals of morbidity scores Bars show the frequency distribution of known morbid genes (in percent) per 0.2 intervals of normal morbidity scores.The blue-shaded area represents the frequency distribution of known morbid genes (in percent) per intervals of shuffled morbidity scores.

and druggability datasets without one of the features, which we call "without-one-feature" datasets as described in "Methods". We then compared the output AUC values with those of meta-classifier trained on datasets with all features by using the Wilcoxon signed-rank statistical test [6]. A difference is considered statistically significant If the obtained $W$ is lower than or equal to $W_{c}$ with a given $N$ at the $\mathrm{p}=0.05$ level (see "Methods"). Note that we use AUC instead of recall or precision to compare the overall performances of meta-classifiers because it represents the meta-classifier performance across all combinations of recall and precision (see "Methods"). Table 2 shows that the median AUC of our meta-classifier trained on morbidity datasets without the number of tissues in which the gene is expressed at least 5 transcripts per million (tpm) (see "Methods" for details) was statistically lower than the median AUC for normal morbidity datasets ( $W=7$ versus $W_{c}=8$ for $N=10$ at $p$ $=0.05)$. So, the tissue expression profile seems to be an important feature to distinguish morbids from non-morbids genes.

As shown in Table 3, for prediction of druggable genes, the overall performance (AUC) of our meta-classifier was statistically lower following the removal of the plasma membrane feature $\left(W=1\right.$ versus $W_{c}=8$ for $N=$ 10 at $p=0.05)$. This result is in concert with the most important cellular rule for druggability derived from the analysis of decision trees (see more details in "Methods") that we will show in the section "Cellular rules for gene morbidity and druggability"): if proteins are located in plasma membrane, their encoding genes are likely to be druggable. This rule is supported by Bakheet and Doig [8] that demonstrated that proteins encoded by druggable genes had more transmembrane helices than proteins encoded by non-druggable ones which suggests that proteins encoded by druggable genes are more likely to be found in plasma membrane.

\section{Comparison with other methods}

Regarding prediction of morbid genes, there have been several methods available for predicting morbid genes [9-16]. However, our method can not be directly compared to most of them since they have been constructed to predict only small sets of disease-specific candidate genes, such as ENDEAVOUR [13] and ToppGene [15], while our method has been constructed for the genomewide prediction of morbid genes. We can, however, compare our method to PROSPECTR [9], CIPHER [14] 


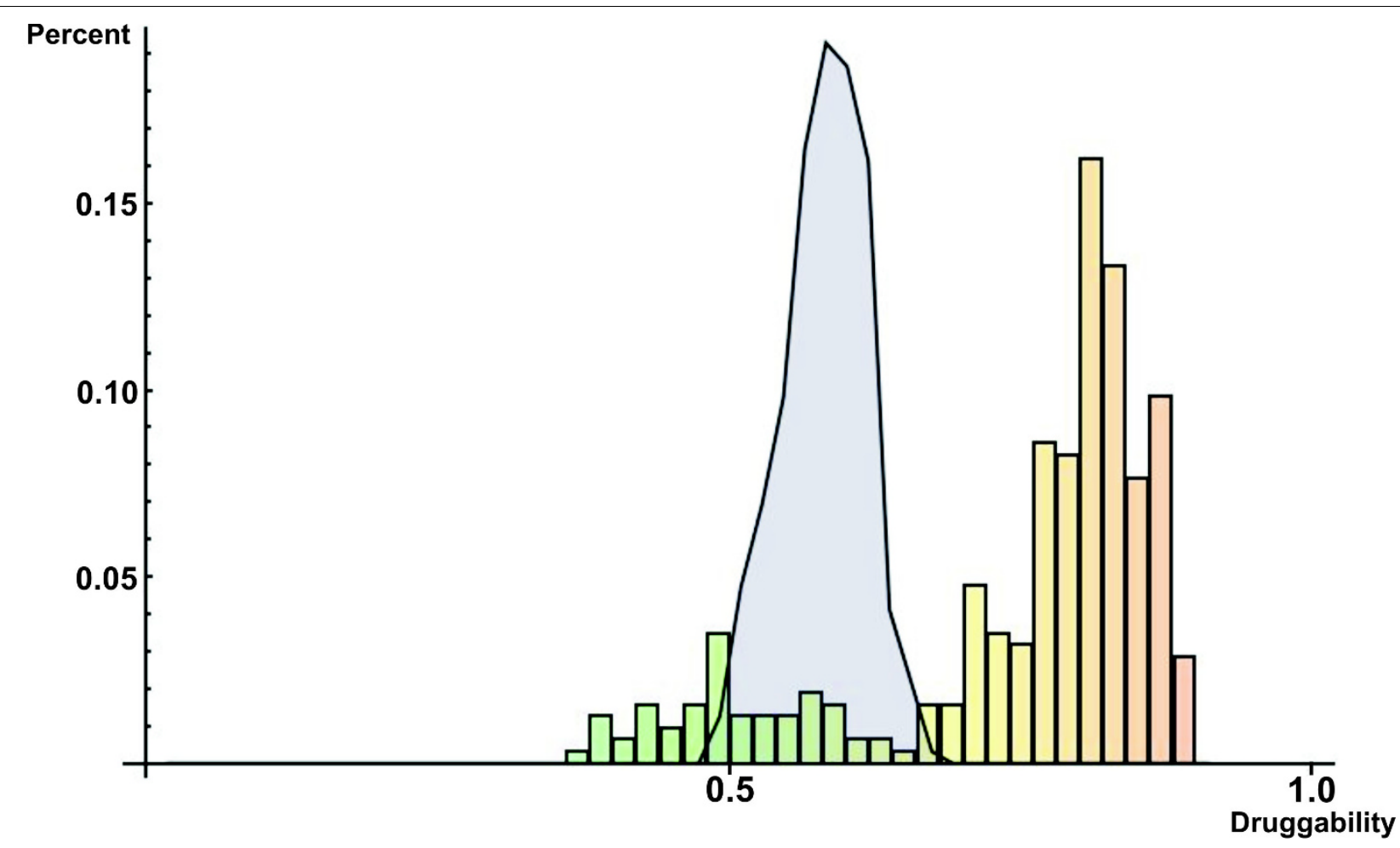

Figure 2 Frequency distribution of known druggable genes per intervals of druggability scores Bars show the frequency distribution of known druggable genes (in percent) per 0.2 intervals of normal druggability scores.The blue-shaded area represents the frequency distribution of known druggable genes (in percent) per intervals of shuffled druggability scores

and that developed by $\mathrm{Xu}$ and $\mathrm{Li}[16]$. Our method outperforms CIPHER (this method, for genome-wide prediction, yields a precision of about 0.1 ; there is no value of recall reported) and is comparable to PROSPECTR that achieves a recall of 0.70 , a precision of 0.62 and an AUC of 0.70. Although PROSPECTR has a higher recall, we considered our method comparable to it as the precision and AUC values of our method are higher than those of PROSPECTR. Moreover, our performance measures are medians of 10 runs of 10 -cross-fold validation (see "Methods" for more details), while the performance measures of PROSPECTR were obtained by only one run of 10-cross-fold validation.

The method developed by $\mathrm{Xu}$ and $\mathrm{Li}$ is the only genome-wide prediction method that apparently outperforms our method (this method achieves, for genomewide prediction, an average recall about 0.78 and an average precision about 0.77 ). Their method is also based on network topological parameters, but while we trained our meta-classifier on various features, including 12 network topological parameters (see "Methods" and Additional file 1), they trained their classifiers on only five network topological parameters: degree, defined as the number of links to node $i ; 1 \mathrm{~N}$ index, defined as the proportion of the number of links to morbid genes among all links to node $i$; $2 \mathrm{~N}$ index, defined as the proportion of the number of links to morbid genes among all links to neighbors of node $i$; the average distance to morbid genes; and positive topological coefficient, a variant of the classical topological coefficient [17]. The apparent success of $\mathrm{Xu}$ and $\mathrm{Li}$ approach in predicting morbid genes mostly relies on the $2 \mathrm{~N}$ index: when node $i$ is a morbid gene, $2 \mathrm{~N}$ index is always higher than zero since at least one neighbor of node $i$ 's neighbor-the node $i$ itself-is a morbid gene; if node $i$ is a non-morbid gene, $2 \mathrm{~N}$ index is higher than or equal to zero. Thus, this parameter induces a spurious correlation on dataset that is captured by classifiers that, in turn, achieve high performance measures. Therefore, the $\mathrm{Xu}$ and Li method can be disregarded for comparison purposes and, accordingly, our approach, although showing moderate recall and precision values, is currently, along with PROSPECTR, the most accurate predictor of morbid genes on a genome-wide scale.

Concerning the prediction of druggable genes, as for prediction of morbid genes, we can compare our method only with those developed to predict druggable genes on a genome-wide scale. Therefore, to our knowledge, we can compare our methodology with that developed by Sugaya and Ikeda [18]. Using support vector 
Table 2 Statistical comparison of performances of classifiers trained on normal and without-one-feature morbidity datasets

\begin{tabular}{|c|c|c|c|c|}
\hline Missing feature $^{1}$ & Median AUC $[\min , \max ]^{2}$ & N & W & $W_{c}$ (two-tailed $\left.p=0.05\right)^{3}$ \\
\hline ppi & $0.715[0.705,0.726]$ & 10 & 26 & 8 \\
\hline metin & $0.714[0.707,0.727]$ & 10 & 26 & 8 \\
\hline metout & $0.713[0.707,0.729]$ & 10 & 25 & 8 \\
\hline regin & $0.714[0.703,0.726]$ & 9 & 18 & 6 \\
\hline regout & $0.716[0.705,0.729]$ & 10 & 26 & 10 \\
\hline c & $0.713[0.701,0.724]$ & 10 & 13 & 8 \\
\hline identicalness & $0.711[0.704,0.727]$ & 10 & 24 & 8 \\
\hline cent & $0.714[0.707,0.727]$ & 10 & 25 & 8 \\
\hline inbet & $0.716[0.708,0.731]$ & 10 & 25 & 8 \\
\hline inbetppi & $0.714[0.707,0.727]$ & 9 & 21 & 6 \\
\hline inbetmet & $0.714[0.707,0.728]$ & 9 & 21 & 6 \\
\hline inbetreg & $0.715[0.706,0.727]$ & 10 & 25 & 8 \\
\hline numtissuesexp ${ }^{4}$ & $0.709[0.701,0.719]$ & 10 & 7 & $8^{*}$ \\
\hline avegexptec ${ }^{5}$ & $0.715[0.704,0.727]$ & 10 & 27 & 8 \\
\hline Unknown & $0.713[0.701,0.725]$ & 10 & 18 & 8 \\
\hline Cytoplasm & $0.715[0.706,0.728]$ & 10 & 26 & 8 \\
\hline Endoplasmic reticulum & $0.716[0.705,0.727]$ & 10 & 26 & 8 \\
\hline Mitochondrion & $0.714[0.706,0.728]$ & 10 & 24 & 8 \\
\hline Nucleus & $0.715[0.704,0.728]$ & 10 & 24 & 8 \\
\hline Other localization & $0.714[0.704,0.726]$ & 10 & 21 & 8 \\
\hline Cellular component & $0.714[0.705,0.727]$ & 9 & 21 & 6 \\
\hline Extracellular space & $0.710[0.7,0.723]$ & 10 & 14 & 8 \\
\hline Golgi apparatus & $0.715[0.706,0.728]$ & 10 & 26 & 8 \\
\hline
\end{tabular}

Median AUC [min,max] for normal datasets: 0.716 [0.706,0.729]

\footnotetext{
1 See "Methods" and Additional file 1 for a description of features

2 Of 10 datasets

${ }^{3}$ According to table of critical values for $W$ in [6]

${ }^{4}$ The number of tissues (out of 32) in which the gene is expressed at least 5 transcripts per million (tpm) according to Reverter et al. [33]

${ }^{5}$ The average expression in tpm among all the tissues in which the gene is expressed according to Reverter et al. [33]

* Difference statistically significant
}

machines trained on 69 different features covering structural, drug and chemical, and functional information on protein-protein interactions, Sugaya and Ikeda classifiers achieved an average recall of $75 \%$, an average precision of $70 \%$ and an average AUC of $72 \%$, performance measures comparable to those obtained by our metaclassifier.

\section{Prediction of novel morbid and druggable genes}

Since the morbidity and druggability of most of genes in INHGI are unknown-only $\approx 14 \%$ and $\approx 3 \%$ are known to be morbid and druggable, respectively-we applied our trained meta-classifier to determine the morbidity and druggability statuses of these genes. Instead of simply predicting genes as morbid or druggable, we decided to assign a "morbidity score" and a "druggability score" (see "Methods") to each gene since we understand that there is no gene that is absolutely non-morbid or nondruggable. We also assigned to each gene a "shuffled morbidity score" and a "shuffled druggability score" to test the significance of normal scores. For this purpose, we used the Wilcoxon signed-rank statistical test as described in "Methods".

Table 4 shows genes not known to be morbid with the 10 highest morbidity scores (see Additional file 2 for the normal and shuffled morbidity scores of all genes in INHGI). All these scores are significantly higher than the shuffled scores ( $W \leq W_{c}$ with $N=10$ at the $\mathrm{p}=$ 0.05 level; see "Methods" and [6]). With the purpose of investigating whether the assigned scores resemble the potential morbidities of these genes, we mined the Human Genome Epidemiology Network (HuGENet) database [19] for articles clearly stating that such genes may be associated with some disease, which we call as "morbidity evidences". According to this approach, we found that 10 of $11(\approx 90 \%)$ genes with the 10 highest morbidity scores are considered to be associated with some disease (Table 4). This shows that our meta-classifier is quite capable of assigning high morbidity scores to genes potentially morbid. 
Table 3 Statistical comparison of performances of classifiers trained on normal and without-one-feature druggability datasets

\begin{tabular}{|c|c|c|c|c|}
\hline Missing feature ${ }^{1}$ & $\begin{array}{l}\text { Median AUC } \\
{[\text { min, } \max ]^{2}}\end{array}$ & $N$ & $W$ & $\begin{array}{c}W_{c} \text { (two-tailed } \\
p=0.05)^{3}\end{array}$ \\
\hline ppi & $0.819[0.798,0.835]$ & 10 & 27 & 8 \\
\hline metin & $0.817[0.803,0.834]$ & 10 & 26 & 8 \\
\hline metout & $0.817[0.801,0.832]$ & 9 & 20 & 6 \\
\hline regin & $0.818[0.799,0.83]$ & 9 & 18 & 6 \\
\hline regout & $0.818[0.801,0.833]$ & 10 & 26 & 8 \\
\hline c & $0.821[0.799,0.836]$ & 10 & 21 & 8 \\
\hline identicalness & $0.819[0.8,0.836]$ & 10 & 27 & 8 \\
\hline cent & $0.814[0.797,0.832]$ & 10 & 18 & 8 \\
\hline inbet & $0.821[0.804,0.837]$ & 10 & 25 & 8 \\
\hline inbetppi & $0.819[0.803,0.833]$ & 10 & 25 & 8 \\
\hline inbetmet & $0.82[0.791,0.833]$ & 10 & 26 & 8 \\
\hline inbetreg & $0.818[0.802,0.83]$ & 9 & 19 & 6 \\
\hline numtissuesexp ${ }^{4}$ & $0.806[0.795,0.832]$ & 9 & 11 & 6 \\
\hline avegexptec $^{5}$ & $0.814[0.799,0.835]$ & 10 & 23 & 8 \\
\hline Unknown & $0.816[0.796,0.832]$ & 9 & 12 & 6 \\
\hline Cytoplasm & $0.814[0.794,0.834]$ & 10 & 20 & 8 \\
\hline $\begin{array}{l}\text { Endoplasmic } \\
\text { reticulum }\end{array}$ & $0.820[0.799,0.834]$ & 10 & 27 & 8 \\
\hline Mitochondrion & $0.820[0.796,0.831]$ & 9 & 22 & 6 \\
\hline Nucleus & $0.816[0.793,0.831]$ & 10 & 20 & 8 \\
\hline Other localization & $0.821[0.802,0.837]$ & 9 & 20 & 6 \\
\hline $\begin{array}{l}\text { Cellular } \\
\text { component }\end{array}$ & $0.82[0.801,0.835]$ & 10 & 25 & 8 \\
\hline Extracellular space & $0.817[0.8,0.837]$ & 10 & 26 & 8 \\
\hline Golgi apparatus & $0.812[0.8,0.834]$ & 10 & 24 & 8 \\
\hline Plasma membrane & $0.781[0.762,0.816]$ & 10 & 1 & $8^{*}$ \\
\hline
\end{tabular}

Median AUC [min,max] for normal datasets : 0.820 [0.801,0.835]

"See "Methods" and Additional file 1 for a description of features

2 Of 10 datasets

${ }^{3}$ According to table of critical values for $W$ in [6]

${ }^{4}$ The number of tissues (out of 32) in which the gene is expressed at least 5 transcripts per million (tpm) according to Reverter et al. [33]

${ }^{5}$ The average expression in tpm among all the tissues in which the gene is expressed according to Reverter et al. [33]

* Difference statistically significant

Table 5 shows genes not known to be druggable with the 10 highest druggability scores (see Additional file 2 for the normal and shuffled druggability scores of all genes in INHGI). All these scores are significantly higher than the shuffled scores ( $W \leq W_{c}$ with $N=10$ at the $\mathrm{p}=$ 0.05 level; see "Methods" and [6]). With the purpose of investigating whether the assigned scores resemble the potential druggabilities of these genes, we mined the literature for articles clearly stating that such genes may be drug target candidates, which we call as "druggability evidences". According to this approach, we found that 8 of $11(\approx 73 \%)$ genes with the 10 highest druggability scores are considered to be drug target candidates (Table 5). This shows that our meta-classifier is quite capable of assigning high druggability scores to genes potentially druggable. Among these candidates, five ( $P L A U, C D 8 A$, $C D 19, I T G A M$ and IL6) are known morbid genes and two (THBS1 and TIMP2) are within the list of genes with the 10 highest morbidity scores. About the known morbid genes with druggability evidence-PLAU, CD19, $I T G A M$ and $I L 6-$, it is interesting to note that the druggabilities assigned to these genes by our classifier are not related to the diseases caused by their corresponding mutated versions. The gene PLAU is a susceptibility gene for late-onset Alzheimer disease according to the Online Mendelian Inheritance in Man (OMIM) database [20] (MIM \# 191840), but the protein encoded by this gene seems to be a good candidate target for treatment of cancer in combination with conventional therapeutics such as chemotherapy or radiation [21]. Similarly, mutations in the gene $C D 19$ cause antibody deficiency that increases susceptibility to infection ([22] (MIM \#107265), but its encoded protein has proven to be a promise as a novel and well-tolerated therapy in B-cell non-Hodgkin's lymphoma [23]. Regarding ITGAM, while Yang et al.[24] have confirmed the association of the this gene with disease susceptibility and renal nephritis of systemic lupus erythematosus (MIM \# 609939), Romano et al.[25], on the other hand, have suggested that the protein encoded by ITGAM is a potential target of the femtomolar-acting eight-amino-acid peptide for protection against the deleterious effects of closed head injury in mice. Finally, according to OMIM database (MIM \# 147620), the gene IL6 mediates growth failure in Crohn disease [26], but we found that its encoded protein is a promising target for therapy of several chronic inflammatory and autoimmune diseases as well as in cancer [27]. These findings show that our classifier, besides discovering new druggable genes, can also reveal unexpectedly roles for known morbid genes in the modulation of diseases caused by other seemingly unrelated genes.

Two potential morbid genes, THBS1 and TIMP2, reinforce the fact that our meta-classifier is able to reveal unexpectedly roles for morbid genes in the modulation of diseases caused by other seemingly unrelated genes. Mutations in the gene THBS1 have been suggested to play a role in atherosclerosis and thrombosis [28], but its encoded protein may be considered a promising therapeutic target for diabetic nephropathy [29]; alterations in TIMP2 has been demonstrated to be one of the causes of chronic obstructive pulmonary disease [30], but targeting its encoded protein may be a therapeutic intervention against connective amino acid tissue degradation [30].

\section{Cellular rules for gene morbidity and druggability}

Beyond the prediction capability, machine learning techniques can be used for knowledge acquisition in order to 
Table $\mathbf{4}$ List of the human genes in the INHGI with the $\mathbf{1 0}$ highest morbidity scores

\begin{tabular}{|c|c|c|c|c|c|c|}
\hline Gene & Morbidity score & $(\text { Median }[\text { min,max }])^{1}$ & $N$ & W & $W_{c}^{2}($ two-tailed $p=0.05)$ & Morbidity evidence ${ }^{3}$ \\
\hline & Normal & Shuffled & & & & \\
\hline TFRC & $0.880[0.576,0.939]$ & $0.568[0.447,0.678]$ & 10 & 1 & $8^{*}$ & 5941956 \\
\hline ITGA5 & $0.875[0.635,0.916]$ & $0.491[0.377,0.631]$ & 10 & 0 & $8^{*}$ & No evidence \\
\hline LTF & $0.868[0.803,0.913]$ & $0.509[0.356,0.642]$ & 10 & 0 & $8^{*}$ & 19258923 \\
\hline SFTPD & $0.866[0.618,0.923]$ & $0.565[0.458,0.682]$ & 10 & 2 & $8^{*}$ & 19590686 \\
\hline THBS1 & $0.865[0.831,0.918]$ & $0.511[0.354,0.566]$ & 10 & 0 & $8^{*}$ & 18178577 \\
\hline TIMP2 & $0.860[0.603,0.92]$ & $0.574[0.388,0.609]$ & 10 & 0 & $8^{*}$ & 19933216 \\
\hline TGFB2 & $0.857[0.565,0.918]$ & $0.526[0.407,0.707]$ & 10 & 3 & $8^{*}$ & 19258923 \\
\hline CGA & $0.856[0.62,0.916]$ & $0.535[0.283,0.656]$ & 10 & 0 & $8^{*}$ & 19730683 \\
\hline SPP1 & $0.856[0.577,0.887]$ & $0.564[0.34,0.696]$ & 10 & 0 & $8^{*}$ & 15868370 \\
\hline FLT1 & $0.854[0.61,0.931]$ & $0.527[0.424,0.715]$ & 10 & 3 & $8^{*}$ & 19741061 \\
\hline NOL3 & $0.850[0.647,0.875]$ & $0.576[0.31,0.651]$ & 10 & 1 & $8^{*}$ & 19773279 \\
\hline
\end{tabular}

${ }^{1}$ Of 10 scores

${ }^{2}$ According to table of critical values for $W$ in [6]

${ }^{3}$ Pudmed IDs of most recent article(s) clearly stating a gene-disease association

* Difference statistically significant

describe patterns in datasets. The machine learning algorithms most used for knowledge acquisition are those that generate decision trees. Decision trees are decision support tools inferred from the training data that use a graph of conditions and their possible consequences. The structure of a decision tree consists of a root node representing the most important condition for discriminating classes, internal nodes representing additional conditions for class discrimination under the main condition, and leaf nodes representing the final classification. So, one can learn the conditions for classifying instances in a given class by following the path from the root node to the leaf node [31].
Therefore, in order to discover the rules for gene morbidity and druggability, we analyzed decision trees generated by training the J48 algorithm, a WEKA's implementation of the C4.5 algorithm [32] (for more details, see "Methods"), on the normal morbidity and druggability datasets containing all network topological features, tissue expression profiles and subcellular localization as training data. The decision trees in Figures 3 and 4 are the best representative tree among the 10 generated decision trees for morbidity (Figure 3 ) and the 10 generated decision trees for druggability (Figure 4).

From the best representative decision tree for morbidity, we were able to devise some general rules for

Table $\mathbf{5}$ List of the human genes in the INHGI with the $\mathbf{1 0}$ highest druggability scores

\begin{tabular}{|c|c|c|c|c|c|c|}
\hline Gene & Druggability score & $(\text { Median }[\min , \max ])^{1}$ & $N$ & $W$ & $W_{c}^{2}$ (two-tailed $p=0.05$ ) & Druggability evidence $^{3}$ \\
\hline & Normal & Shuffled & & & & \\
\hline $\mathrm{HLA}-\mathrm{F}$ & $0.887[0.803,0.915]$ & $0.530[0.427,0.584]$ & 10 & 0 & $8^{*}$ & No evidence \\
\hline $\mathrm{PLAU}^{4}$ & $0.886[0.808,0.907]$ & $0.561[0.387,0.675]$ & 10 & 0 & $8^{*}$ & 19301652 \\
\hline $\mathrm{CD}_{8} \mathrm{~A}^{4}$ & $0.885[0.871,0.902]$ & $0.56[0.37,0.664]$ & 10 & 0 & $8^{*}$ & No evidence \\
\hline CD19 4 & $0.880[0.751,0.907]$ & $0.562[0.38,0.628]$ & 10 & 0 & $8^{*}$ & 19509168 \\
\hline ITGAM $^{4}$ & $0.878[0.614,0.887]$ & $0.534[0.36,0.656]$ & 10 & 1 & $8^{*}$ & 11931348 \\
\hline $\mathrm{THBS}^{5}$ & $0.875[0.53,0.9]$ & $0.532[0.293,0.592]$ & 10 & 0 & $8^{*}$ & 17878288 \\
\hline ITGAX & $0.873[0.784,0.897]$ & $0.539[0.422,0.691]$ & 10 & 0 & $8^{*}$ & No evidence \\
\hline CXCR5 & $0.871[0.755,0.895]$ & $0.537[0.49,0.59]$ & 10 & 0 & $8^{*}$ & 17652619 \\
\hline EBI3 & $0.871[0.801,0.888]$ & $0.529[0.391,0.626]$ & 10 & 0 & $8^{*}$ & 19556516 \\
\hline $1 \mathrm{~L} 6^{4}$ & $0.87[0.766,0.893]$ & $0.591[0.361,0.643]$ & 10 & 0 & $8^{*}$ & 17465721 \\
\hline TIMP2 $2^{5}$ & $0.869[0.645,0.916]$ & $0.584[0.34,0.701]$ & 10 & 0 & $8^{*}$ & 10985804 \\
\hline
\end{tabular}

\footnotetext{
${ }^{1}$ Of 10 scores

${ }^{2}$ According to table of critical values for $W$ in [6]

${ }^{3}$ Pudmed IDs of most recent articles clearly stating that such genes may be drug target candidates

${ }^{4}$ Morbid genes according to Morbid Map [46]

${ }^{5}$ Genes among those with 10 highest morbidity scores (Table 4)

* Difference statistically significant
} 


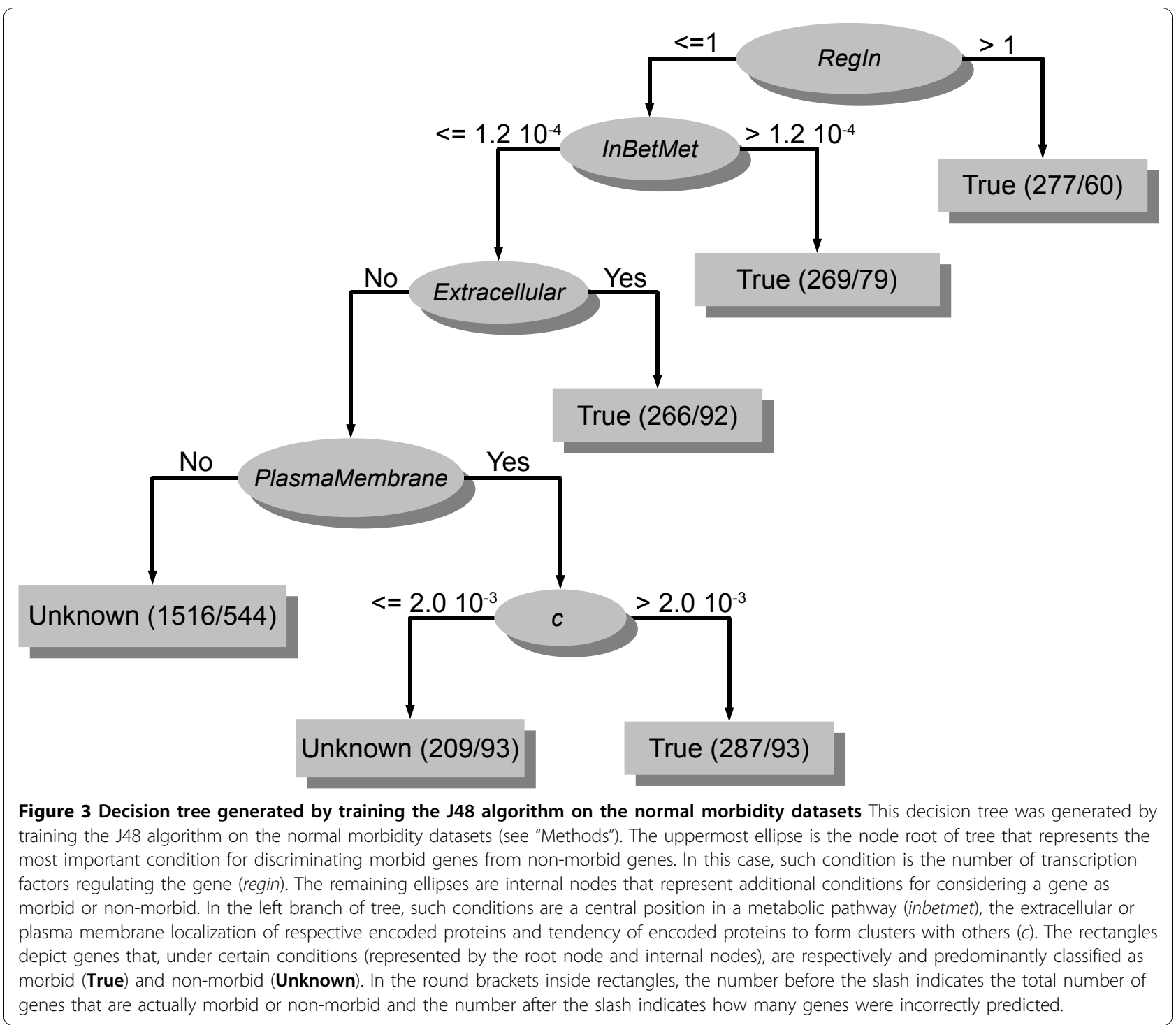

morbidity in human. As we can observe in Figure 3, the root node of decision tree is the number of transcription factors that regulate a given gene (regin). So, this attribute can be considered the most important feature, among those used to train the $\mathrm{J} 48$ algorithm, for discriminating a morbid from a non-morbid gene. To reinforce this, we found, by walking the path from root node to first leaf node through the right branch, the following rule for morbidity: if genes are regulated by more than one transcription factor, they are likely to be morbid (Figure 3). The study by Reverter et al.[33] supports this rule as they showed that morbid genes are more likely to show tissue specific expression than nonmorbid ones. Genes whose expression is tissue specific tend to be regulated by more transcription factors than those that are ubiquitously expressed, e.g. housekeeping genes, since a high level of transcriptional regulation is needed in this case.

Walking the path from root node to first and second leaf nodes through the left branch (Figure 4), we found the following rule for morbidity: if genes are regulated by one transcription factor and their encoded proteins are either centrally located in metabolic pathways (inbetmet is the betweenness centrality via metabolic interactions; see "Methods" and Additional file 1) or play a role in the extracellular region, genes are likely to be morbid. This rule is supported by Jimenez-Sanchez and colleagues [34] that showed that morbid genes are more likely to be enzymes than non-morbid ones and by Winter et al. [35] that demonstrated that $\approx 40 \%$ of proteins encoded by morbid genes are predicted to be secreted. Furthermore, if proteins are neither centrally located in 


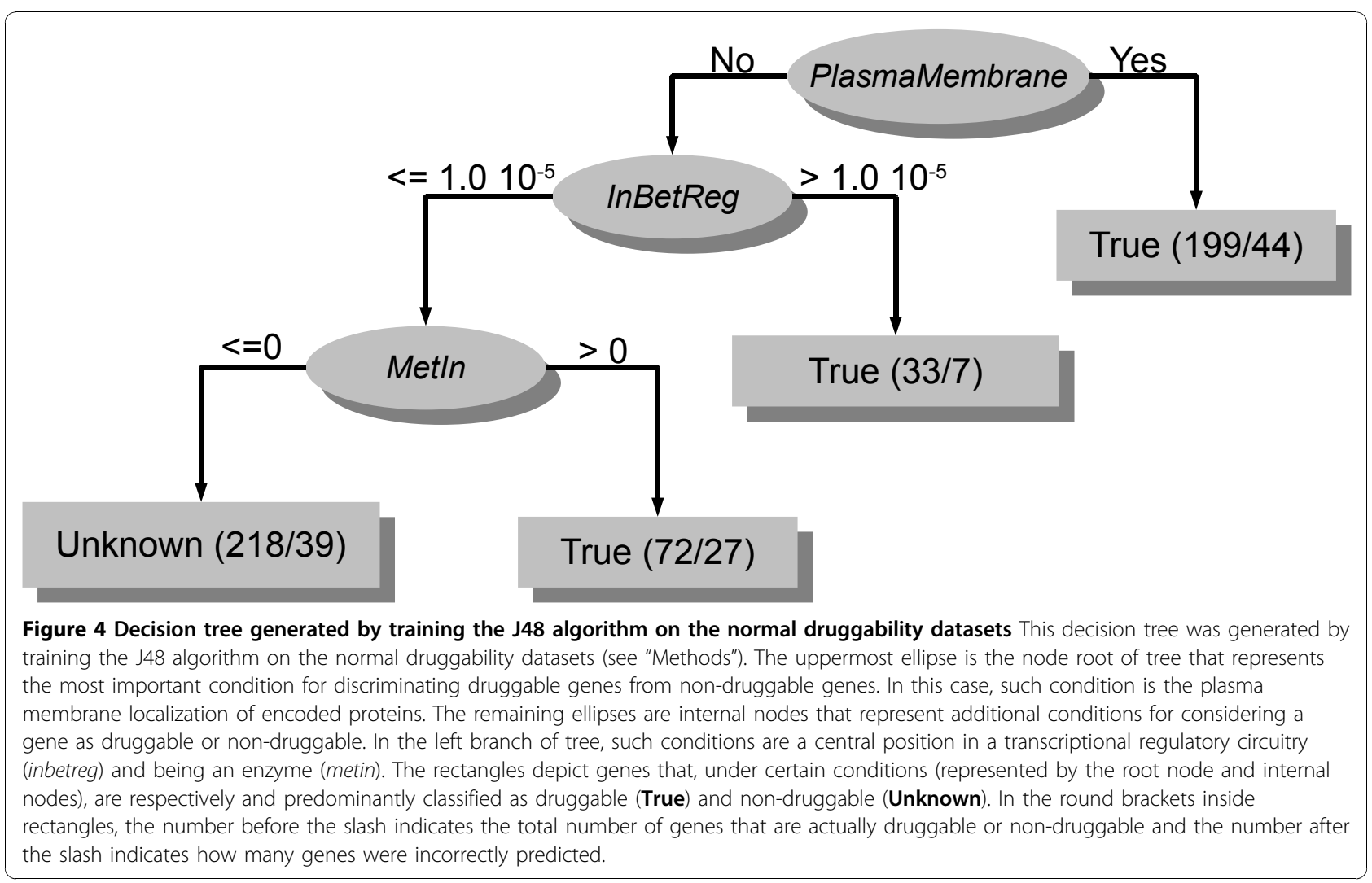

metabolic pathways nor play a role in the extracellular region but are located in plasma membrane and tend to form clusters with other proteins (recall that $c$ is the clustering coefficient, a network feature the measures the local group cohesiveness; see "Methods" and Additional file 1), their encoding genes are likely to be morbid. For this rule, we could not find any article supporting it. Therefore, the plasma membrane localization of proteins encoded by morbid genes as well as the tendency of these proteins to form clusters with other proteins are issues to be examined.

From the best representative decision tree for druggability, we were able to devise some general rules for druggability in human. As we can observe in Figure 4, the root node of decision tree is the plasma membrane localization of proteins. So, this attribute can be considered the most important feature, among those used to train the J48 algorithm, for discriminating a druggable from a non-druggable gene. To reinforce this, we found, by walking the path from root node to first leaf node through the right branch, the following rule for druggability: if proteins are located in plasma membrane, their encoding genes are likely to be druggable (Figure 4). This rule is supported by Bakheet and Doig [8] that demonstrated that proteins encoded by druggable genes had more transmembrane helices than proteins encoded by non-druggable ones which suggests that proteins encoded by druggable genes are more likely to be found in plasma membrane. Walking the path from root node to first and second leaf nodes through the left branch (Figure 4), we found the following rule for druggability: if proteins are not located in plasma membrane but are either centrally located in a transcriptional regulatory circuitry (inbetreg is the betweenness centrality via transcriptional regulation interactions; see "Methods" and Additional file 1) or are enzymes (metin is the number of metabolites catalyzed by a given enzyme; see Additional file 1), their encoding genes are likely to be druggable. This rule is partially supported by Bakheet and Doig [8] as they showed that druggable proteins are more likely to be enzymes than non-morbid ones. In respect to central position in a transcriptional regulatory circuitry, this is an issue that remains to be elucidated.

\section{Conclusions}

The identification of morbid and druggable genes has largely been an experimental effort mostly performed by time-consuming experiments. In an effort to accelerate the pace of discovery of such genes, we designed a machine learning-based computational approach that relies on network topological features, tissue expression profile and subcellular localization information for predicting morbid and druggable genes in human on a genome-wide scale. 
We could demonstrate that our method is able to reliable predict morbid and druggable genes on a genome-wide scale as demonstrated by (i) the moderate to high performance measures achieved by the meta-classifiers (Table 1), (ii) the observation that the designed meta-classifiers learned traits actually related to morbidity and druggability instead of traits associated with any random sets of genes (Table 1) and (iii) the fact that known morbid and druggable genes tend to have high morbidity and druggability scores, respectively (Figures 1 and 2). Furthermore, in comparison with other available genome-wide prediction methods, the performance of our method proved to be equal or superior. We could also devise some cellular rules for gene morbidity and druggability using all network topological features, tissue expression profile and subcellular localization information as learning attributes for generation of decision trees (see details in section "Cellular rules for gene morbidity and druggability"). We discovered that number of regulating transcription factors, the central position in metabolic pathways, the localization of their encoded proteins in extracellular region and plasma membrane and tendency to form clusters with other proteins are important factors determining gene morbidity. In respect to druggability, the important factors determining druggability are plasma membrane localization, a central position in a transcriptional regulatory circuitry and being an enzyme. The fact that almost all discovered rules are supported by some additional evidences solidifies decision trees as useful tools for extracting knowledge from complex biological data. Albeit the good prediction performance and the ability to discover cellular rules for morbidity and druggability, our approach suffers from three limitations. First, it depends on existing Gene Ontology annotation and interaction data which are likely to be enriched in small-scale experiments involving morbid and druggable genes. Second, the construction of an integrated network of gene interactions requires a large amount of experimental interaction data that are currently available only to a limited number of human genes-our INHGI, for example, covers only $\approx 25 \%$ of already identified human genes. Third, the lack of negative examples to train the classifier forces us to consider all genes not known to be morbid or druggable as de facto non-morbid and non-druggable genes. We expect, however, that such limitations will be soon addressed as more systems-level data are generated.

\section{Methods}

Generation of the set of training features Network topological features

In order to compute the network topological features used as training features for predicting morbid and druggable genes, we first constructed an integrated network of human gene interactions (INHGI) based on assumption that two genes, $g_{1}$ and $g_{2}$, coding respectively for proteins $p_{1}$ and $p_{2}$, are interacting genes if (i) $p_{1}$ and $p_{2}$ interact physically (protein physical interaction), (ii) the transcription factor $p_{1}$ directly regulates the transcription of gene $g_{2}$, i.e., $p_{1}$ binds to the promoter region of $g_{2}$ (transcriptional regulation interaction), or (iii) the enzymes $p_{1}$ and $p_{2}$ share metabolites, i.e., a product generated by a reaction catalyzed by enzyme $p_{1}$ is used as reactant by a reaction catalyzed by enzyme $p_{2}$ (metabolic interaction). Experimentally verified human protein physical interactions data were obtained from the following databases: the Biological General Repository for Interaction Datasets (BioGRID) database (release 2.0.47; [36]), the Database of Interacting Proteins (DIP; release Hsapi20081014; [37]), the Human Protein Reference Database (HPRD; release 7; [1]), IntAct (release 91; [38]), the Molecular Interactions Database (MINT; October 2008 release; [39]) and The Munich Information Center for Protein Sequences (MIPS) Mammalian Protein Interaction Database (MPPI; downloaded in December 2008; [40]. Experimentally verified human transcriptional regulation interactions were obtained from the Transcriptional Regulatory Element Database (TRED; [41]).

Experimentally verified human metabolic interactions were extracted from the human metabolic model Recon 1 [42] by a code implemented in Mathematica ${ }^{\circ}$ 7.0 (Wolfram Research, Inc.). We excluded those metabolic interactions generated by the so-called "currency metabolites", abundant molecular species present throughout the cell most of the time and, therefore, unlikely to impose any constraints on the dynamics of metabolic reactions. Due to this feature of currency metabolites, the functionality of the network would be better represented without them [43]. We considered currency metabolites the eight most connected metabolites (ADP, ATP, $\mathrm{H}+, \mathrm{H}_{2} \mathrm{O}, \mathrm{NADP}+, \mathrm{NADPH}$, orthophosphate and pyrophosphate) in the original metabolic model Recon 1.

The final INHGI is the result of integration of the protein physical, metabolic and transcriptional regulation interactions datasets through genes common to these datasets. Before performing the integration, we converted all human gene names to their GeneIDs-as provided by the Entrez Gene database [5] - to avoid the creation of false interactions due to gene name ambiguity.

For each gene $g$ in INHGI, we computed 12 network topological features as listed in Additional file 1. Briefly, degree centrality is defined as the number of links to node (in our case, gene). We considered each type of interaction as a distinct measure of degree as described 
in Additional file 1. Clustering coefficient $(c)$ of a node (in our case, a gene) quantifies how close the node and its neighbors are to being a clique, i.e., all nodes connected to all nodes. For the INHGI, $c$ is defined as the proportion of links between the genes within the neighborhood of $g$ divided by the number of links that could possibly exist between them. Betweenness centrality reflects the role played by a node (in our case, a gene) in the global network architecture and, for the INHGI, is defined as the fraction of shortest paths between $g_{i}$ and $g_{j}$ passing through $g$. We computed the betweenness centrality based on shortest paths via all types of interaction (inbet) as well as based on shortest paths via each type of interaction (inbetppi, inbetmet and inbetreg). Closeness centrality (cent) measures how close a node (in our case, a gene) is to all others in the network and, for the INHGI, is defined as the mean shortest path between $g$ and all other genes reachable from it. Identicalness is the number of genes with identical network topological characteristics.

All these network topological features, except for the betweenness centrality-related features, were calculated by a program written in a Mathematica 7.0 notebook. The betweenness centrality-related features were calculated by the Python package NetworkX 0.99 [44].

\section{Subcellular localization of human genes}

We determined the subcellular localization of proteins encoded by the genes in the INHGI by using the QuickGO tool, a Gene Ontology (GO) browser associated with the integrated database resource for protein families (InterProt) at the European Bioinformatics Institute [45]. We selected GO slim terms-subsets of GO terms consisting of a limited number of high-level GO terms that cover some or all of the content of GOrelated to cellular components provided by QuickGO to annotate genes in the INHGI. Genes were annotated to the following slim terms:"cytoplasm", "endoplasmic reticulum", "mitochondrion", "nucleus", "extracellular space", "Golgi apparatus", "plasma membrane" and "cellular component". Genes annotated to other slim terms were reannotated to one of these terms or to a new term named "other localization" and genes with no GO cellular component slim term annotation was annotated to the term "unknown".

\section{Tissue expression profile of human genes}

We retrieved the tissue expression profiles of genes in the INHGI from the study performed by Reverter and colleagues [33]. In their study, Reverter and colleagues mined three large datasets comprising expression data obtained from massively parallel signature sequencing across 32 tissues in order to classify genes as housekeeping or tissue-specific genes and then relate this tissue specificity with gene interactions and disease states. According to Reverter and colleagues, tissue expression profile of a given gene is $(i)$ the number of tissues (out of 32) in which the gene is expressed at least 5 transcripts per million (tpm) and (ii) the average expression in tpm among all the tissues in which the gene is expressed [33].

\section{Classifier design and evaluation \\ Construction of training datasets}

For evaluating the performance of the chosen training features-network topological features, subcellular localization and tissue expression profile-in predicting morbid and druggable genes, we constructed four different groups of balanced training datasets, i.e., datasets containing the same number of positive (in our case, morbid or druggable genes) and negative (in our case, nonmorbid or non-druggable genes) examples: (1) "normal morbidity datasets", (2) "shuffled morbidity datasets", (3) "normal druggability datasets" and (4) "shuffled druggability datasets".

For the construction of the morbidity datasets, we first gathered a list of "morbid genes"-genes whose mutations cause hereditary diseases-from the morbid map table in the Online Mendelian Inheritance in Man (OMIM) [46] and then mapped them to the INHGI. The final list of morbid genes used as positive examples to train our classifier is comprised by 1,412 morbid genes present in the INHGI. Regarding the negative examples, we considered as "non-morbid genes" the remaining genes present in the INHGI; this was done since building a list of genes not known to be involved in hereditary diseases is impossible currently. We randomly selected 10 different sets of 1,412 of these nonmorbid genes and combine them with the list of morbid genes to build 10 different training datasets which we call "normal morbidity datasets". From these normal morbidity datasets, we generate 10 different "shuffled morbidity datasets" by randomly shuffling the class labels (morbid and non-morbid) among genes.

For the construction of the druggability dataset, we first built a list of "druggable genes"-genes coding for proteins whose modulation by small molecules elicits phenotypic effects-from the drug-target network constructed by Yildirim and colleagues [47] and then mapped them to the INHGI. The final list of druggable genes used as positive examples to train our classifier is comprised by 257 druggable genes present in the INHGI. Regarding the negative examples, we considered as "non-druggable genes" the remaining genes present in the INHGI; this was done since, similar to non-morbid genes, it is also impossible to construct a list of genes coding for proteins whose modulation by small molecules do not elicits phenotypic effects. We randomly selected 10 different sets of 257 of these nondruggable genes and combine them with the list of 
druggable genes to build 10 different training datasets which we call "normal druggability datasets". From these normal druggability datasets, we generate 10 different "shuffled druggability datasets" by randomly shuffling the class labels (druggable and non-druggable) among genes. We also constructed 25 additional morbidity and 25 additional druggability datasets lacking one of the 25 features used as training attributes. We call these datasets as "without-one-feature" datasets, where one can be replaced by the name of feature.

\section{Classifier design}

Using WEKA (Waikato Environment for Knowledge Analysis) software package, a collection of machine learning algorithms for data mining tasks [48], we designed the classifier used for predicting morbid and druggable genes in the INHGI. This classifier is an ensemble of seven decision tree algorithms using the meta-classifier "Vote", a WEKA's implementation of the voting algorithm that combines the output predictions of each classifier by different rules [49]. We combined the classifiers by the average rule, where the output predictions computed by the individual classifiers for each class are averaged and this average is used in its decision [49]. The classifiers composing our model were: (1) REPtree [48], (2) random tree [48], (3) random forest [50], (4) J48, a WEKA's implementation of the C4.5 decision tree [32], with minimum number of 32 instances per leaf, (5) best-first decision tree with minimum number of 32 instances at the terminal nodes [51], (6) logistic model tree [52] and (7) alternating decision tree with 25 boost iterations [53]. In addition, we applied the bootstrap aggregating (bagging) approach [54] to each classifier. Parameters values for each classifier are provided in the Additional file 3.

\section{Classifier evaluation}

We assessed the performance of our classifier by estimating the following measures: recall, precision and area under the curve (AUC) of the receiver operating characteristic (ROC) curve. Recall is the proportion of actual morbid or druggable genes which are correctly predicted as such against all actual morbid or druggable genes:

$$
\text { Recall }=\frac{T P}{T P+F N}
$$

TP (true positive) denotes the amount of actual morbid or druggable genes correctly predicted as such and FN (false negative) denotes the amount of actual morbid or druggable genes incorrectly predicted as non-morbid or non-druggable, respectively.

Precision is the proportion of actual morbid or druggable genes which are correctly predicted as such against all genes predicted as morbid or druggable:

$$
\text { Precision }=\frac{T P}{T P+F P}
$$

FP denotes the amount of actual non-morbid or nondruggable genes incorrectly predicted as morbid or druggable, respectively.

The AUC is a widely used summary measure of the ROC curve-a plot of the true positive rate versus false positive rate that indicates the probability of a true positive prediction as a function of the probability of a false positive prediction for all possible threshold values [55]-and is equivalent to the probability that a randomly chosen negative example (in our case, a non-morbid or non-druggable gene) will have a smaller estimated probability of belonging to the positive class than a randomly chosen positive example (in our case, a morbid or druggable gene) [56].

We estimated the above-mentioned performance measures by performing a 10 -fold cross-validation testusing WEKA-on the 10 normal and 10 shuffled morbidity datasets and on the 10 normal and 10 shuffled druggability datasets constructed as described in the section "Construction of training datasets". During the 10fold cross-validation test process, each dataset is randomly partitioned into 10 subsets. Of the 10 subsets, a single subset is retained as the validation data for testing the model, and the remaining 9 subsets are used as training data. The cross-validation process is then repeated 10 times, with each of the 10 subsets used exactly once as the validation data. The 10 results from the folds are then averaged to produce a single estimation for each performance measure for each dataset. We reported the performance measures estimated by the 10 -fold cross-validation as medians of the 10 datasets for each category (normal morbidity, shuffled morbidity, normal morbidity and shuffled morbidity).

The statistical comparisons of (i) the performance measures estimated by our classifier trained on normal and shuffled datasets, (ii) the AUC values estimated by our classifier trained on normal datasets and withoutone-feature datasets, and (iii) the normal and shuffled morbidity and druggability scores for each gene in INHGI were performed by the Wilcoxon signed-rank test [6]. Following established conventions in the machine learning community, we used this test since it makes minimal assumptions about the underlying distribution of performance measures used to evaluate classifiers [57]. The differences were statistically significant if the obtained Wilcoxon's test statistic value $(W)$ was equal to or smaller than the critical Wilcoxon's test statistic value $\left(W_{c}\right)$ for a given sample size $(N)$ at the twotailed significance level of $0.05(p=0.05)$ according to the table of critical values for the Wilcoxon test [6]. 


\section{Prediction of novel morbid and druggable genes}

The "normal morbidity scores" and the "normal druggability scores" were generated by applying the models constructed by training our meta-classifier on the normal datasets to the entire set of genes in INHGI where the class labels were removed. These scores are the probability values of classifying each gene as morbid or druggable as returned by the models. The final normal morbidity and druggability scores are median scores of 10 scores. We also obtained "shuffled morbidity scores" and "shuffled druggability scores" that were generated by models trained on the shuffled datasets.

\section{Determination of rules for gene morbidity and druggability}

The determination of rules for gene morbidity and druggability was performed by analyzing the best representative decision tree for each category among the 10 decision trees generated through the training of $\mathrm{J} 48$ algorithm [32] on the 10 normal morbidity and 10 normal druggability datasets. The parameters values for producing decision trees by $\mathrm{J} 48$ algorithm training are provided in the Additional file 3.

\section{Additional material}

Additional file 1: Network topological features Description: This file includes a table showing the functions and descriptions of the 12 network topological features used as learning attributes for training the classifier algorithm

Additional file 2: Morbidity and druggability scores of genes in INHGI Description: Tab-limited text file containing all genes (Entrez GenelDs) in the INHGI with their morbidity and druggability scores.

Additional file 3: Parameters used to train the meta-classifier and J48 Description: File containing all parameters values used to train the meta-classifier for prediction of morbid and druggable genes and all parameters values used to train the $\mathbf{J} 48$ algorithm to generate decision trees for discovery of cellular rules for morbidity and druggability.

\section{Competing interests statement}

The authors declare that they have no competing interests.

\begin{abstract}
Acknowledgments
The authors would like to thank FAPESP (The State of Sao Paulo Research Foundation) for the financial support through the FAPESP research grants 2007/02827-9, 2007/01213-7 and 2007/08466-8. This research was supported by resources supplied by the Center for Scientific Computing (NCC/ GridUNESP) of the Univ Estadual Paulista (UNESP).

This article has been published as part of BMC Genomics Volume 11 Supplement 5, 2010: Proceedings of the 5th International Conference of the Brazilian Association for Bioinformatics and Computational Biology. The full contents of the supplement are available online at http://www. biomedcentral.com/1471-2164/11?issue=S5
\end{abstract}

\section{Authors contributions}

PRC obtained the tissue expression profile and gene ontology data, analyzed the meta-classifiers' performances, implemented the program for calculation of network topological features and drafted the manuscript. MLA obtained all interaction data, constructed the network, designed the meta-classifier, pursued the biological interpretation of results and drafted the manuscript. $\mathrm{NL}$ conceived, designed and directed the project. All authors read and approved the final manuscript.

Published: 22 December 2010

\section{References}

1. Keshava Prasad TS, Goel R, Kandasamy K, Keerthikumar S, Kumar S, Mathivanan S, Telikicherla D, Raju R, Shafreen B, Venugopal A, Balakrishnan L, Marimuthu A, Banerjee S, Somanathan DS, Sebastian A, Rani S, Ray S, Harrys Kishore CJ, Kanth S, Ahmed M, Kashyap MK, Mohmood R, Ramachandra YL, Krishna V, Rahiman BA, Mohan S, Ranganathan P, Ramabadran S, Chaerkady R, Pandey A: Human Protein Reference Database-2009 update. Nucleic Acids Res 2009, 37(Database issue):D767-72.

2. Lindsay MA: Target discovery. Nat Rev Drug Discov 2003, 2(10):831-8.

3. da Silva JPM, Acencio ML, Mombachb JCM, Vieirac R, da Silva J, Lemke N, Sinigagliac M: In silico network topology-based prediction of gene essentiality. Physica A 2008, 387:1049-1055.

4. Acencio ML, Lemke $\mathrm{N}$ : Towards the prediction of essential genes by integration of network topology, cellular localization and biological process information. BMC Bioinformatics 2009, 10:290.

5. Maglott D, Ostell J, Pruitt KD, Tatusova T: Entrez Gene: gene-centered information at NCBI. Nucleic Acids Res 2007, 35:D26-D31.

6. Wilcoxon F: Probability tables for individual comparisons by ranking methods. Biometrics 1947, 3(3):119-22.

7. Stumpf MPH, Thorne T, de Silva E, Stewart R, An HJ, Lappe M, Wiuf C: Estimating the size of the human interactome. Proc Natl Acad Sci U S A 2008, 105(19):6959-64.

8. Bakheet TM, Doig AJ: Properties and identification of human protein drug targets. Bioinformatics 2009, 25(4):451-7.

9. Adie EA, Adams RR, Evans KL, Porteous DJ, Pickard BS: Speeding disease gene discovery by sequence based candidate prioritization. BMC Bioinformatics 2005, 6:55.

10. Perez-Iratxeta $C$, Bork $P$, Andrade MA: Association of genes to genetically inherited diseases using data mining. Nat Genet 2002, 31(3):316-9.

11. Turner FS, Clutterbuck DR, Semple CAM: POCUS: mining genomic sequence annotation to predict disease genes. Genome Biol 2003, 4(11): R75.

12. Van Driel MA, Cuelenaere K, Kemmeren PPCW, Leunissen JAM, Brunner HG: A new web-based data mining tool for the identification of candidate genes for human genetic disorders. Eur J Hum Genet 2003, 11:57-63.

13. Aerts $S$, Lambrechts $D$, Maity $S$, Van Loo P, Coessens B, De Smet F, Tranchevent LC, De-Moor B, Marynen P, Hassan B, Carmeliet P, Moreau Y: Gene prioritization through genomic data fusion. Nat Biotechnol 2006, 24(5):537-44.

14. Wu X, Jiang R, Zhang MQ, Li S: Network-based global inference of human disease genes. Mol Syst Biol 2008, 4:189.

15. Chen J, Bardes EE, Aronow BJ, Jegga AG: ToppGene Suite for gene list enrichment analysis and candidate gene prioritization. Nucleic Acids Res 2009, 37(Web Server issue):W305-11.

16. $\mathrm{Xu} \mathrm{J,} \mathrm{Li} \mathrm{Y:} \mathrm{Discovering} \mathrm{disease-genes} \mathrm{by} \mathrm{topological} \mathrm{features} \mathrm{in} \mathrm{human}$ protein-protein interaction network. Bioinformatics 2006, 22(22):2800-5.

17. Goldberg DS, Roth FP: Assessing experimentally derived interactions in a small world. Proc Natl Acad Sci U S A 2003, 100(8):4372-6.

18. Sugaya $N$, Ikeda K: Assessing the druggability of protein-protein interactions by a supervised machine-learning method. BMC Bioinformatics 2009, 10:263.

19. Lin BK, Clyne M, Walsh M, Gomez O, Yu W, Gwinn M, Khoury MJ: Tracking the epidemiology of human genes in the literature: the HuGE Published Literature database. Am J Epidemiol 2006, 164:1-4.

20. Finckh U, van Hadeln K, Müller-Thomsen T, Alberici A, Binetti G, Hock C, Nitsch RM, Stoppe G, Reiss J, Gal A: Association of late-onset Alzheimer disease with a genotype of PLAU, the gene encoding urokinase-type plasminogen activator on chromosome 10q22.2. Neurogenetics 2003, 4(4):213-7.

21. Gondi CS, Rao JS: Therapeutic potential of siRNA-mediated targeting of urokinase plasminogen activator, its receptor, and matrix metalloproteinases. Methods Mol Biol 2009, 487:267-81.

22. van Zelm MC, Reisli I, van der Burg M, Castaño D, van Noesel CJM, van Tol MJD, Woellner C, Grimbacher B, Patiño PJ, van Dongen JJM, Franco JL: 
An antibody-deficiency syndrome due to mutations in the CD19 gene. $N$ Engl J Med 2006, 354(18):1901-12.

23. Al-Katib AM, Aboukameel A, Mohammad R, Bissery MC, Zuany-Amorim C: Superior antitumor activity of SAR3419 to rituximab in xenograft models for non-Hodgkin's lymphoma. Clin Cancer Res 2009, 15(12):4038-45.

24. Yang W, Zhao M, Hirankarn N, Lau CS, Mok CC, Chan TM, Wong RWS, Lee KW, Mok MY, Wong SN, Avihingsanon Y, Lin IO, Lee TL, Ho MHK, Lee PPW, Wong WHS, Sham PC, Lau YL: ITGAM is associated with disease susceptibility and renal nephritis of systemic lupus erythematosus in Hong Kong Chinese and Thai. Hum Mol Genet 2009, 18(11):2063-70.

25. Romano J, Beni-Adani L, Nissenbaum OL, Brenneman DE, Shohami E, Gozes I: A single administration of the peptide NAP induces long-term protective changes against the consequences of head injury: gene Atlas array analysis. J Mol Neurosci 2002, 18(1-2):37-45.

26. Sawczenko A, Azooz O, Paraszczuk J, Idestrom M, Croft NM, Savage MO, Ballinger $A B$, Sanderson IR: Intestinal inflammation-induced growth retardation acts through IL-6 in rats and depends on the -174 IL- 6 G/C polymorphism in children. Proc Natl Acad Sci U S A 2005, 102(37):13260-5.

27. Rose-John S, Waetzig GH, Scheller J, GrÖtzinger J, Seegert D: The IL-6/sIL$6 \mathrm{R}$ complex as a novel target for therapeutic approaches. Expert Opin Ther Targets 2007, 11(5):613-24.

28. Koch W, Hoppmann P, de Waha A, SchÖmig A, Kastrati A: Polymorphisms in thrombospondin genes and myocardial infarction: a case-control study and a meta-analysis of available evidence. Hum Mol Genet 2008, 17(8):1120-6.

29. Daniel C, Schaub K, Amann K, Lawler J, Hugo C: Thrombospondin-1 is an endogenous activator of TGF-beta in experimental diabetic nephropathy in vivo. Diabetes 2007, 56(12):2982-9.

30. Castaldi PJ, Cho MH, Cohn M, Langerman F, Moran S, Tarragona N, Moukhachen H, Venugopal R, Hasimja D, Kao E, Wallace B, Hersh CP, Bagade S, Bertram L, Silverman EK, Trikalinos TA: The COPD genetic association compendium: a comprehensive online database of COPD genetic associations. Hum Mol Genet 2010, 19(3):526-34.

31. Kingsford C, Salzberg SL: What are decision trees? Nat Biotechnol 2008, 26(9):1011-1013.

32. Quinlan JR: C4.5: programs for machine learning. San Francisco: Morgan Kaufmann; 1993.

33. Reverter A, Ingham A, Dalrymple B: Mining tissue specificity, gene connectivity and disease association to reveal a set of genes that modify the action of disease causing genes. BioData Min. 2008, 1:8.

34. Jimenez-Sanchez G, Childs B, Valle D: Human disease genes. Nature 2001, 409(6822):853-5.

35. Winter EE, Goodstadt L, Ponting CP: Elevated rates of protein secretion, evolution, and disease among tissue-specific genes. Genome Res 2004, 14:54-61.

36. Breitkreutz BJ, Stark C, Reguly T, Boucher L, Breitkreutz A, Livstone M, Oughtred R, Lackner DH, Bähler J, Wood V, Dolinski K, Tyers M: The BioGRID Interaction Database: 2008 update. Nucleic Acids Res 2008, 36(Database issue):D637-40.

37. Salwinski L, Miller CS, Smith AJ, Pettit FK, Bowie JU, Eisenberg D: The Database of Interacting Proteins: 2004 update. Nucleic Acids Res 2004, 32(Database issue):D449-51.

38. Hermjakob H, Montecchi-Palazzi L, Lewington C, Mudali S, Kerrien S, Orchard S, Vingron M, Roechert B, Roepstorff P, Valencia A, Margalit $H$, Armstrong J, Bairoch A, Cesareni G, Sherman D, Apweiler R: IntAct: an open source molecular interaction database. Nucleic Acids Research 2004, 32:D452-D455.

39. Chatr-aryamontri A, Ceol A, Palazzi LM, Nardelli G, Schneider MV, Castagnoli L, Cesareni G: MINT: the Molecular INTeraction database. Nucleic Acids Res. 2007, 35:D572-D574.

40. Pagel P, Kovac S, Oesterheld M, Brauner B, Dunger-Kaltenbach I, Frishman G, Montrone C, Mark P, Stümpflen V, Mewes HW, Ruepp A, Frishman D: The MIPS mammalian protein-protein interaction database. Bioinformatics 2005, 21:832-834.

41. Jiang C, Xuan Z, Zhao F, Zhang MQ: TRED: a transcriptional regulatory element database, new entries and other development. Nucleic Acids Res. 2007, 35:D137-D140.

42. Duarte NC, Becker SA, Jamshidi N, Thiele I, Mo ML, Vo TD, Srivas R, Palsson B: Global reconstruction of the human metabolic network based on genomic and bibliomic data. PNAS 2007, 104:1777-1782.
43. Huss M, Holme P: Currency and commodity metabolites: their identification and relation to the modularity of metabolic networks. IET Syst Biol 2007, 1(5):280-285.

44. NetworkX package. [https://networkx.lanl.gov].

45. Binns D, Dimmer E, Huntley R, Barrell D, O'Donovan C, Apweiler R: QuickGO: a web-based tool for Gene Ontology searching. Bioinformatics 2009, 25(22):3045-6.

46. McKusick VA: Mendelian Inheritance in Man and its online version, OMIM. Am J Hum Genet 2007, 80(4):588-604.

47. Yildirim MA, Goh Kl, Cusick ME, Barabási AL, Vidal M: Drug-target network. Nat Biotechnol 2007, 25(10):1119-26.

48. Witten IH, Frank E: Data Mining: Practical Machine Learning Tools and Techniques with Java Implementations. San Francisco: Morgan Kaufmann; 2000.

49. Kittler J, Hatef M, Duin RP, Matas J: On Combining Classifiers. IEEE Trans Pattern Anal Mach Intell. 1998, 20(3):226-239.

50. Breiman L: Random forests. Mach Learn 2001, 45:5-32

51. Shi H: Best-first Decision Tree Learning. Master Thesis The University of Waikato; 2007.

52. Landwehr N, Hall M, Frank E: Logistic Model Trees. Mach Learn 2005, 95(12):161-205.

53. Freund $Y$, Mason $L$ : The alternating decision tree learning algorithm. Proceedings of the Sixteenth International Conference on Machine Learning San Francisco: Morgan Kaufmann; 1999, 124-133.

54. Breiman L: Bagging predictors. Mach Learn 1996, 24(2):123.

55. Huang J, Ling CX: Using AUC and Accuracy in Evaluating Learning Algorithms. IEEE Trans. on Knowl. and Data Eng 2005, 17(3):299-310.

56. Hand DJ, Till RJ: A Simple Generalisation of the Area Under the ROC Curve for Multiple Class Classification Problems. Mach. Learn 2001, 45(2):171-186

57. Demšar J: Statistical Comparisons of Classifiers over Multiple Data Sets. J. Mach. Learn. Res. 2006, 7:1-30

\section{doi:10.1186/1471-2164-11-S5-S9}

Cite this article as: Costa et al:: A machine learning approach for genome-wide prediction of morbid and druggable human genes based on systems-level data. BMC Genomics 2010 11(Suppl 5):S9.

\section{Submit your next manuscript to BioMed Central and take full advantage of:}

- Convenient online submission

- Thorough peer review

- No space constraints or color figure charges

- Immediate publication on acceptance

- Inclusion in PubMed, CAS, Scopus and Google Scholar

- Research which is freely available for redistribution

Submit your manuscript at www.biomedcentral.com/submit
C BioMed Central 